\title{
Modelo metodológico de diseño de sistemas producto-servicio (M.M.SPS) en el contexto empresarial colombiano. Una investigación desde el diseño
}

\begin{abstract}
Resumen
La noción de sostenibilidad surge al afrontar las consecuencias de los impactos ambientales ocasionados por la acelerada industrialización. Su reto consiste en cambiar el fin de la economía liberal: la utilidad por un equilibrio entre las variables sociales, ambientales y económicas. Entre las soluciones exploradas, la disciplina del diseño desarrolló el concepto de sistemas producto-servicio (SPS), modelo de negocios no tradicionales que buscan proporcionar valor ambiental a través del ecodiseño, valor económico con configuraciones innovadoras de redes y valor social diseñando para el usuario. La investigación reconoce en los SPS una poderosa opción para transitar hacia la sostenibilidad y generar competitividad, razón por la cual se han puesto en práctica en varios países europeos; no obstante, de acuerdo con las Naciones Unidas, su implementación es baja en países de Latinoamérica debido a la ausencia de conocimiento y tecnológica, a la falta de metodologías de diseño, a la carencia de capacidades de emprendimiento, experiencia en la gestión de servicios y un marco legal de apoyo (UNEP, 2002).
\end{abstract}

La presente investigación busca generar un aporte desde el diseño para sobrepasar estas barreras de implementación, mediante el desarrollo de una metodología que posibilite la creación de sistemas productoservicio en el contexto colombiano. El enfoque metodológico utilizado es cualitativo, y se seleccionó el método de caso de estudio tipo múltiple.

El resultado principal de la investigación es el Modelo Metodológico de Aproximación a los sistemas producto-servicio (M.M.SPS), mientras que entre las conclusiones se identifican una brecha en conocimientos y capacidades de diseño, innovación, sostenibilidad y gerencia para desarrollar soluciones SPS; un alto potencial de contribución del diseño a través de nuevos roles del diseñador y herramientas de comunicación, visualización e interacción, y la necesidad de optimizar la capacidad de configurar redes empresariales que posibiliten los SPS.

\author{
Nancy Mahecha Lagos \\ Doctor en Diseño y Creación \\ Docente Universidad Externado de \\ Colombia \\ Correo electrónico: \\ nancy.mahecha@uexternado.edu.co \\ ำ orcid.org/0000-0003-0244-1419 \\ Google Scholar
}

Alejandro Boada Ortiz Doctor en Administración de Empresas

Director del Centro de Tecnología y Producción CTP

Universidad Externado de Colombia. Bogotá, Colombia

Correo electrónico:

alejandro.boada@uexternado.edu.co ๑ orcid.org/0000-0003-2261-8293

Google Scholar

Recibido: abril 30 de 2018

Aprobado: septiembre 28 de 2019

\section{Palabras clave:}

Caso de estudio, economía circular, metodologías de diseño, sistemas producto-servicio, sostenibilidad.

Revista KEPES Año 17 No. 21 enero-julio 2020, págs.459-492 ISSN: 1794-7111(Impreso) ISSN: 2462-8115 (En línea) DOI: $10.17151 /$ kepes.2020.17.21.16 (c) (1) 


\title{
Methodological Model of Product-Service System Design (M.M.SPS) in the Colombian Business Context. A research from Design
}

\begin{abstract}
The notion of sustainability arises when facing the consequences of the environmental impacts caused by accelerated industrialization. Its challenge is to change the objective of the liberal economy: profit for a balance between social, environmental and economic variables. Among the solutions explored, the Design discipline developed the concept of Product Service Systems (PSS), a non-traditional business model that seeks to provide environmental value through eco-design, economic value with innovative network configurations and social value designing for the user. This esearch recognizes in SPS a powerful option to move towards sustainability and generate competitiveness, which is why it has been implemented in several European countries. However, according to the United Nations, its implementation is low in Latin American countries due to the lack of design methodologies, the lack of entrepreneurial skills, experience in service management and a legal support framework (UNEP 2002).

This research seeks to generate a contribution from Design to overcome these implementation barriers, developing a methodology that enables the creation of product service systems in the Colombian Context. The methodological approach used is qualitative, and the multiple case study method was selected. The main result of the research is the Methodological Model of Approach to the Product-Service System (M.M.SPS) while among the conclusions, a gap in knowledge and capabilities of design, environmental management and management to develop SPS solutions in emerging countries is identified, as well as a high potential for design contribution through new designer roles, communication, visualization and interaction tools and the need to optimize the ability to configure business networks that enable PSS.
\end{abstract}

Key words:

Circular economy, design methodologies, productservice systems, case study, sustainability. 


\section{Introducción}

El principal reto de la sostenibilidad consiste en cambiar el fin último que propone el sistema de economía liberal, esto es, la utilidad alcanzada a través de la producción para consumo de masas, por un equilibrio entre las variables sociales, ambientales y económicas que posibilite la supervivencia de la humanidad. Este entendimiento de la necesidad de abordar la sostenibilidad de manera integral se confirma por medio de los Objetivos de Desarrollo Sostenible 2030, definidos por las Naciones Unidas; frente al sistema de producción y consumo actual, los países firmantes se comprometen "a efectuar cambios fundamentales en la manera en que nuestras sociedades producen y consumen bienes y servicios" (ONU, 2015).

La comunidad científica y la academia tienen el imperativo de aportar a la construcción y cumplimiento de estos objetivos, en particular la disciplina del diseño, que desempeña un papel fundamental desde su visión holística e integradora y desde su capacidad de realizar transformaciones culturales a partir de la construcción de nuevos valores y significados. Al respecto, Margolin (1995) afirma: "La tarea del diseño es reconceptualizar los valores humanos con el objetivo de crear significados que deriven en grandes posibilidades de satisfacción y la posibilidad de alcanzar una específica calidad de vida deseada" (p. 16).

La sostenibilidad plantea un nuevo concepto de bienestar, el cual implica un cambio radical en lo que la sociedad entiende por calidad de vida; el reto de la disciplina del diseño "recrear" su significado. En términos del diseñador Tony Fry, la tarea del diseñador consiste en la recreación de las condiciones biológicas y sociales necesarias para nuestra supervivencia, es decir, "construir medios de producción que soporten la vida" (Buchanan \& Margolin, 1995, p. 192). 


\section{Diseño y sostenibilidad}

El aporte del diseño a la sostenibilidad se puede entender a través de la expansión de su ámbito de estudio de artefactos físicos a artefactos sociales, y la creación de nuevas áreas de actuación específicas del diseño para el medio ambiente y el valor social.

En lo que tiene que ver con la expansión del ámbito del diseño, son muchos los autores que han reflexionado al respecto; es una realidad, por lo menos teórica, la expansión al campo social y ecológico. ¿Puede el diseño limitarse a productos, utensilios, instalaciones, máquinas, casas, ciudades, o por el contrario es el diseño una decisión sobre una forma de vida? (Aicher, 1992).

Buchanan desde la retórica y Krippendorff desde la semántica plantean la expansión de la disciplina. Buchanan identifica cuatro áreas principales en las que los diseñadores están trabajando actualmente: "diseño gráfico y de comunicaciones visuales, diseño de objetos materiales, diseño de actividades y servicios y, finalmente, el diseño de sistemas complejos, entornos para vivir, trabajar, jugar y aprender, esta última es cada vez más consciente del rol de diseño en la sustentabilidad" (Buchanan, 1992, p. 9).

Por otra parte, Krippendorff (2006) en su propuesta de trayectoria de la artificialidad, plantea seis definiciones de artefactos desde el entendimiento de la evolución de la sociedad y el significado de la interacción individual, social y cultural de los seres humanos: productos, servicios e identidades, interfaces, redes y sistemas multiusuario, proyectos y discursos. En la indagación y entendimiento del artefacto de discursos podemos situar una respuesta a la sostenibilidad, que se define por un tipo de lenguaje propio, el cual construye la realidad y determina las prácticas de los miembros de la comunidad a la que pertenecen. Krippendorff identifica el discurso del diseño; crear metáforas, 
introducir nuevos significados, y hablar diferente son construcciones sociales de mundos alternativos generados en estos artefactos (Krippendorff, 2006) (figura 1).

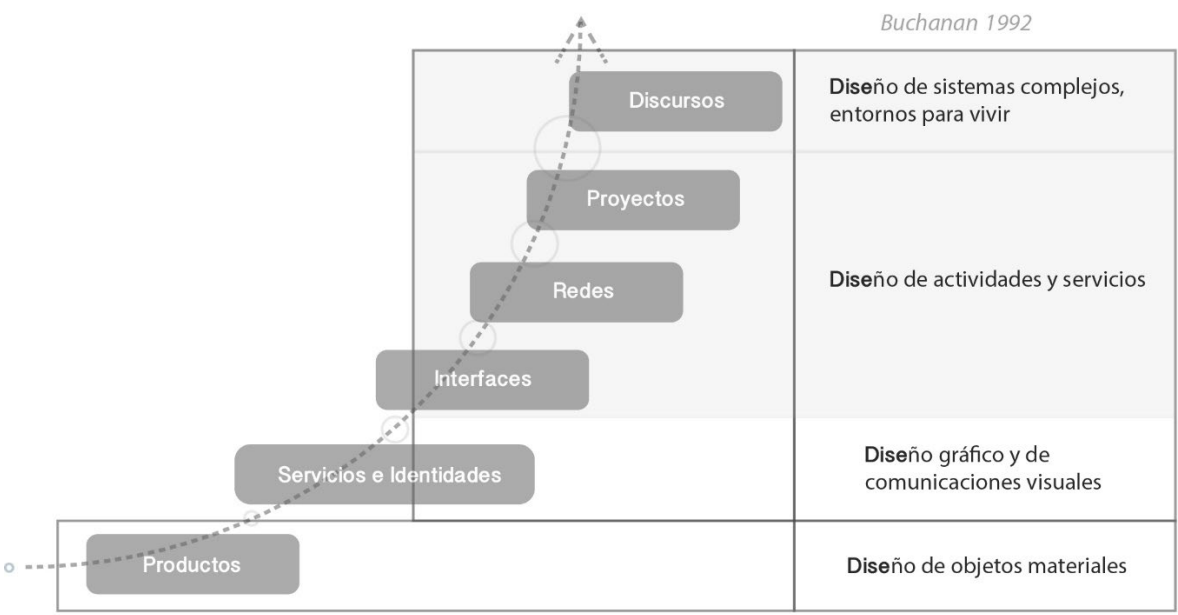

Trayectoria de Artificialidad. (Krippendorff, 2006)

Figura 1. Nuevos ámbitos del diseño. Expansión del área del diseño, acorde con propuestas de Krippendorff (2006) y Buchanan (1992).

En cuanto a la creación de nuevas áreas de actuación del diseño relacionadas con el ambiente y lo social, se identifican cuatro áreas de actuación del diseño en términos de sostenibilidad: el diseño con materiales de bajo impacto y bajo consumo de energía, el ecodiseño, el análisis del ciclo de vida, y el diseño para la equidad y cohesión social, una nueva frontera de investigación.

En la evolución de su aporte a la sostenibilidad, cuando la disciplina del diseño toma en cuenta el comportamiento ambiental de los productos a 
través de decisiones respecto a materiales, consumo de energía y procesos de producción, se habla de un ecodiseño enmarcado en la manufactura de productos. El diseño con enfoque de ciclo de vida amplía la visión tradicional de ecodiseño al abordar la minimización de impactos ambientales en todas las etapas del producto (materiales, producción, distribución, uso y disposición final) y diseñar a partir de la función del producto, ubicándonos en un ámbito más allá del diseño de productos: el diseño funcional.

A pesar de su aporte a la sostenibilidad, el diseñar con enfoque de ciclo de vida ha presentado obstáculos en el sistema tradicional de intercambio comercial; se evidencia una fragmentación entre el ciclo de vida físico de los productos y el flujo de actores en todas las etapas, además de intereses económicos diferentes. Como consecuencia de esto, los diseñadores se han enfrentado a la necesidad de reconfigurar todo el sistema para lograr resultados. Esta situación, unida a la fuerte presión social por conseguir resultados concretos de sostenibilidad, dio origen en los años noventa al concepto de SPS, dentro del diseño estratégico; en este, los diseñadores pasan de diseñar solo productos a diseñar sistemas eficientes, integrando a los actores a lo largo del ciclo de vida de la oferta.

Durante 20 años de investigación, son muchos los conceptos de los sistemas producto-servicio que se han planteado. Goedkoop (1999) los define como una combinación de sistemas y productos capaz de satisfacer totalmente las necesidades de un usuario. Brezet (2001) desde el diseño, asegura que los servicios ecoeficientes son sistemas producto-servicio desarrollados para causar un mínimo impacto ambiental con una generación máxima de valor. El artículo "Clarificando el concepto de SPS", de Mont (2002), se los define como un sistema de productos y servicios soportado en redes e infraestructura, diseñado para ser competitivo, satisfacer las necesidades del usuario y tener un bajo impacto ambiental, menor que los modelos de negocio tradicionales. Para Manzini (2003), son el resultado de una innovación estratégica que cambia el 
énfasis de diseñar y vender productos físicos únicamente a productos y servicios que tienen la capacidad de suplir las demandas específicas de los usuarios. Finalmente, Vezzoli (2014) los presenta desde el diseño como un modelo de oferta que provee e integra productos y servicios que tienen la capacidad de satisfacer la demanda particular de los usuarios, basados en relaciones innovadoras entre los stakeholders del sistema de producción de valor, donde la economía y los intereses de competencia proveen continuamente nuevas soluciones, con beneficios ambientales y sociales.

Las disciplinas en las que las bondades de los sistemas producto-servicio son más evidentes son aquellas en las que se ha enfocado la investigación: diseño, servicios, modelo de negocios, manufactura, sostenibilidad, cadena de suministro y tecnología.

En su recopilación y análisis del concepto, Vasantha (2012) plantea los elementos que intervienen en una solución SPS: oferta integrada (productoservicio), modelo de negocios, valor de innovación adicional (económico, social, ambiental), partes interesadas, entorno, enfoque en ciclo de vida y evaluación de criterios (Vasantha, Rajkumar, \& Lelah, 2012).

Los sistemas producto-servicio son soluciones integradas de productos y servicios que buscan satisfacer las necesidades de un usuario a través de su función (uso), más que de un producto; por lo tanto, tienen un enfoque predominante de servicio (diseño de oferta integrada); su implementación obliga a generar redes de trabajo entre diferentes empresas y actores, para lo cual requiere una estructura, basada en tecnología escalable y flexible (diseño de redes). Debido a su nivel de desmaterialización y forma de consumo diferente de la tradicional (renta, leasing, pago por uso), son soluciones con alto potencial de beneficios ambientales y sociales, aceptadas globalmente como una respuesta al dilema de la sostenibilidad (diseño de sistema sostenible). 
Las soluciones sistema producto-servicio amplían el ámbito del diseño al diseño de sistemas complejos (Buchanan, 1992), innovación de sistema (Brezet, 2001), diseño de discursos (Krippendorff, 2006) o diseño estratégico (Manzini, 2006).

A partir de la expansión del ámbito del diseño, de las áreas de actuación del diseño para la sostenibilidad y de los elementos que forman el concepto de sistema producto-servicio, se identifican tres dimensiones básicas del diseño de un sistema producto-servicio: diseño de oferta integrada, diseño de redes y diseño del sistema sostenible, las cuales se constituyen en la base teórica para la construcción del modelo metodológico propuesto en esta investigación. A continuación, se muestra la relación entre ámbito del diseño, áreas de actuación y dimensiones de diseño de un SPS (figura 2).

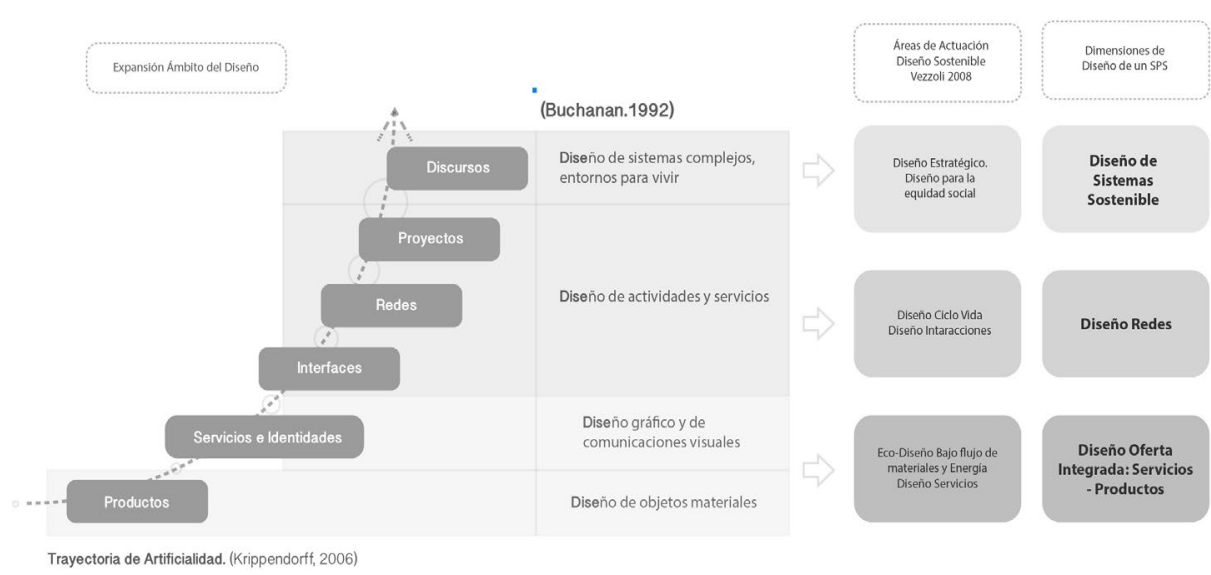

Figura 2. Relación entre expansión del ámbito del diseño, áreas de actuación y dimensiones de diseño un SPS. 


\section{Dimensiones de diseño para una solución SPS}

\section{Dimensión 1. Diseño de oferta integrada.}

Esta dimensión comprende el diseño de una sola solución para que el usuario satisfaga su necesidad a través de tres elementos claves: diseño de producto, diseño de servicio y la integración de estos. El diseñador se enfoca en soluciones conformadas por una mezcla de productos físicos, servicios e interfaces asociados. La puerta de entrada del diseño es la identificación de las necesidades de los usuarios para tipificar la función deseada, y termina en la configuración de una oferta integrada generando servicios innovadores con valor económico, ambiental y social.

Aquí la experticia del diseño es fundamental en el entendimiento del usuario, sus valores, creencias y capacidades para lograr definir soluciones que lo satisfagan y lo reafirmen. El diseñador debe re-crear valores y creencias culturales que permitan generar en el usuario nuevos criterios de calidad y bienestar que conlleven la aceptación de la solución diseñada. Se busca pasar de un consumo de bienes de consumo a un consumo de soluciones que ofrezcan bienestar.

Al ser la dimensión del sistema producto-servicio la que contiene los productos físicos, el diseñador debe buscar eficiencia en términos ambientales con materiales de bajo impacto y bajos consumos de energía. La acción del diseño se realiza a través del ciclo de vida de los productos que se van a diseñar, con el objetivo de disminuir el impacto ambiental y social en cada una de las etapas del producto o servicio: preproducción, producción, distribución, uso y disposición final. 


\section{Dimensión 2. Diseño de redes.}

En esta dimensión hablamos de la configuración de la interacción de las partes interesadas e interfaces de la solución con el usuario. El diseño desempeña un papel vital en la construcción de relaciones de confianza.

El diseño de las redes tiene dos elementos esenciales: entender quiénes son los actores que intervienen a lo largo del flujo de ciclo de vida de la oferta integrada y cuáles son sus intereses, y diseñar interacciones entre estos actores que permitan un máximo valor económico, social y ambiental para las partes. En términos de diseño, es lograr la mejor configuración de las partes para optimizar la función del artefacto sistema producto-servicio.

\section{Dimensión 3. Sistema sostenible: valor ambiental y social.}

Mediante esta dimensión de diseño se busca mover el eje del diseño de productos o servicio a diseño de sistemas sostenibles en el tiempo. Su objetivo consiste en asegurar el valor de la solución en términos económicos, ambientales y sociales.

En esta etapa del diseño se incluyen los criterios y variables deseables de la solución para alcanzar los objetivos relacionados con lo ambiental y social. Se requieren conocimientos y herramientas que permitan medir de manera concreta el valor generado por la solución en cada una de sus fases: diseño, producción, uso y fin de vida. "El proceso de diseño se debe gestionar y orientar hacia las interacciones sostenibles de las partes interesadas" (Vezzoli, Kohtala, \& Srinivasan, 2014). 
Esta investigación encuentra un valioso aporte en la construcción de metodologías de diseño que involucren e interrelacionen de manera eficiente, en la práctica empresarial, las tres dimensiones del diseño antes descritas. Si bien existe una variedad de métodos de diseño de SPS, estos carecen de una crítica evaluación de su funcionamiento tanto en la práctica como en el contexto. Para Boehm,

la investigación de los SPS ha tenido un fuerte enfoque conceptual debido a que los métodos empíricos no son fáciles de llevar a la práctica, incluso métodos como estudios de campo han sido casi dejados de lado; sin embargo, podrían ser muy útiles para comprender mejor el concepto de SPS. (Boehm \& Oliver, 2013, p. 256)

\section{Metodología}

El enfoque metodológico utilizado en la investigación es cualitativo. Se seleccionó el método de caso de estudio del tipo múltiple de diseño incrustado acorde con Yin (2014), caracterizado por buscar una reproducción lógica de los resultados repitiendo la metodología sobre casos diferentes con dos o más unidades de análisis, obteniéndose más pruebas y mejorando la validez externa de la investigación.

El Modelo Metodológico de Diseño de SPS (M.M.SPS) es el resultado de la aproximación metodológica propuesta por la investigación "Construcción teórica de un modelo de diseño de una solución SPS (prototipo) basado en el entendimiento de los factores críticos de implementación y de la adaptación de cuatro metodologías proclives a implementar en Colombia"; validación del prototipo propuesto a través de la conducción de tres casos de estudio, para por último establecer el modelo final a partir de un ejercicio de triangulación de información de casos de estudio-caso deéxito-entrevistas y teórica, consolidándose una constante interacción entre la teoría y el trabajo de campo que permitió contribuciones teóricas que fueron nuevamente validadas en campo. 


\section{Primera fase. Construcción teórica}

La construcción teórica arrojó tres resultados: identificación de factores críticos de implementación (FCI), validación de metodologías de diseño de SPS que se pueden desarrollar en Colombia y prototipo inicial del M.M.SPS.

\section{Identificación de factores críticos de implementación.}

La identificación de los factores críticos de implementación (FCI) partió de la elaboración del estado del arte de los SPS a través de análisis bibliométrico. La información se obtuvo de las bases académicas Scopus y Science Direct, y revista Design Issue. La ecuación de búsqueda utilizada fue ("Product service systems"). Como resultado de esto, se identificaron 844 artículos en el periodo de análisis 2000-2016: 334 (Science Direct), 62 (Design Issue) y 448 en Scopus.

Los autores referentes del tema por citaciones y número de artículos son: Oksana Mont (Suecia), Ezio Manzini (Italia), Brezet (Holanda), Nicola Moreli (Dinamarca).

En una etapa de revisión más detallada para la identificación de factores críticos del total de artículos, se seleccionaron 23 relacionados con factores o barreras de implementación de los SPS y estados del arte: (Goedkoop, 1999), (White, Stoughton, \& Feng, 1999), (UNEP, 2002), (Mont, 2002), (Manzini, 2003), (Boada \& Mont, 2005), (Kanda \& Nakagami, 2006) (Tukker, 2006), (Baines, Lightfoot, \& Steve, 2007), (Tsai \& Hsin, 2010), (Clayton \& Backhouse, 2011), (Vasantha, Rajkumar, \& Lelah, 2012), (Tukker, 2013), (Ceschin, 2013), 
(Boehm \& Oliver, 2013), (Catulli, Lindley \& Reed, 2013), (Hänsch \& Gitirana, 2013), (Laperche \& Picard, 2013), (Pezzotta, Cavalieri, \& Shimomura, 2013), (Schenkl, Rösch, \& Mörtl, 2014), (Vezzoli, Kohtala, \& Srinivasan, 2014), (Vezzoli, Ceschin, Diehl, \& Kohtala, 2015), (Reim, Parida, \& Örtqvist, 2015). A continuación, se hizo una revisión exhaustiva de dichos artículos, gracias a la cual se identificaron los factores recurrentes y más referenciados por los autores como críticos para la implementación de los sistemas producto-servicio. Se identificó un total de 43 factores críticos de implementación. Los factores se integraron por coincidencia de conceptos y actor de las soluciones SPS. De la integración se identificaron 11 factores críticos: enfoque de manufactura, nivel de conocimiento de economía ambiental y SPS, capacidades de gestión y tecnología, cultura de innovación, nivel de riesgo, modelo de negocio, compromiso gerencial, confianza, ausencia política pública, capacidades de diseño y contexto favorable.

\section{Valoración de metodologías factibles de desarrollar en Colombia.}

La identificación de metodologías se desarrolló con los artículos dedicados a metodologías de diseño de sistema producto-servicio. Se eligieron ocho metodologías factibles de implementar en el contexto de Colombia, de un total de 40 encontradas. Los criterios que guiaron al grupo de expertos en la selección de estas metodologías fueron los siguientes: autores principales, enfoque del SPS, relevancia en la bibliografía existente, nivel de utilización de la metodología y nivel de similitud con prácticas empresariales de Colombia (tabla 1). 
Tabla 1. Metodologías de diseño SPS seleccionadas para hacer análisis en profundidad.

\begin{tabular}{|c|c|c|c|}
\hline N. ${ }^{o}$ & Metodología & Autor & Artículo de citación \\
\hline 1 & Método Kathaly & Luiten, 2001 & (Vasantha, Rajkumar, \& Lelah, 2012) \\
\hline 2 & $\begin{array}{l}\text { Diseño de Servicios } \\
\text { Ecoeficientes (DES) }\end{array}$ & Brezet, 2001 & $\begin{array}{l}\text { (Vasantha, Rajkumar, \& Lelah, 2012). } \\
\text { (Clayton \& Backhouse, 2011). } \\
\text { (Tukker, } 2013\end{array}$ \\
\hline 3 & $\begin{array}{l}\text { Soluciones Orientadas al } \\
\text { Partnership (SPO) }\end{array}$ & Ezio Manzini, 2004 & $\begin{array}{l}\text { (Manzini, Solution oriented partnership., } \\
\text { 2004) }\end{array}$ \\
\hline 4 & $\begin{array}{l}\text { How to design } \\
\text { industrialised sustainable } \\
\text { solutions? }\end{array}$ & Rochi, 2005 & (Vezzoli, Kohtala, \& Srinivasan, 2014) \\
\hline 5 & $\begin{array}{l}\text { Diseño de SPS a través } \\
\text { del análisis estratégico } \\
\text { (MEPSS) }\end{array}$ & Van Halen, 2005 & $\begin{array}{l}\text { (Vasantha, Rajkumar, \& Lelah, 2012). } \\
\text { (Clayton \& Backhouse, 2011). } \\
\text { (Tukker, 2013) } \\
\text { (Baines, Lightfoot, \& Steve, 2007) }\end{array}$ \\
\hline 6 & $\begin{array}{l}\text { Desarrollo de } \\
\text { herramientas } \\
\text { metodológicas para } \\
\text { generar soluciones } \\
\text { sistémicas, incluyendo } \\
\text { productos y servicios } \\
\text { (DEP) }\end{array}$ & Morelli, 2002, 2006 & $\begin{array}{l}\text { (Vasantha, Rajkumar, \& Lelah, 2012). } \\
\text { (Clayton \& Backhouse, 2011). } \\
\text { (Tukker, 2013) }\end{array}$ \\
\hline 7 & $\begin{array}{l}\text { Diseño de Sistemas de } \\
\text { Servicio (SSD) }\end{array}$ & Kar, 2010 & (Clayton \& Backhouse, 2011) \\
\hline 8 & $\begin{array}{l}\text { Methodology for System } \\
\text { Design for Sustainability } \\
\text { (MSDS) }\end{array}$ & Vezzoli, 2014 & (Vezzoli, Kohtala, \& Srinivasan, 2014) \\
\hline
\end{tabular}

Nota: Metodologías seleccionadas con el objetivo de identificar las más próximas al contexto de la investigación que soporten la construcción del prototipo metodológico inicial. 
La metodología de valoración consistió en la identificación teórica de tres variables latentes esenciales en la implementación exitosa de una metodología sistema producto-servicio, para cada una de ellas se definieron ítems que posibilitaron su cuantificación:

1. Variable 1. Capacidad de diseño de un sistema producto-servicio: mide el nivel de inclusión en la metodología de los ejes básicos de diseño de un SPS: 1 . diseño de oferta integrada, 2 . diseño de redes y 3 . diseño de sistema sostenible (12 ítems).

2. Variable 2. Grado de factibilidad de implementación: mide la presencia de los ítems claves de implementación de una metodología de SPS en el contexto empresarial colombiano. Variable construida por grupos de expertos en gestión empresarial (ocho ítems).

3. Variable 3. Inclusión de factores críticos de implementación: Valora la presencia de los factores críticos de implementación identificados por la investigación como fundamentales en la aplicación de los SPS en el contexto de estudio (siete ítems).

La valoración se hizo con un grupo de cinco expertos en gestión empresarial, a través de un escalamiento de medida tipo Likert de cuatro puntos relacionados con el impacto de cada ítem evaluado sobre el éxito de implementación de las metodologías sometidas a estudio. Acorde con la escala, las metodologías con el más alto puntaje de calificación son las de mayor ajuste al contexto colombiano en términos de la posibilidad exitosa de implementación.

Cada una de las ocho metodologías preliminares fue evaluada por el grupo de expertos en gestión empresarial, con el objetivo de identificar las de mayor potencial de implementación en el contexto y sobre estas realizar la construcción del prototipo inicial del M.M.SPS. De acuerdo con los resultados arrojados por la escala de medición propuesta, se seleccionaron las metodologías 
Brezet, Vezzoli, MEPSS y Rochi. La metodología más recomendada para el contexto de Colombia es la de Brezet. Esta metodología, originaria del proceso tradicional de diseño de productos, presenta una evolución sencilla hacia los sistemas producto-servicio y se adapta a las capacidades de diseño presentes en el contexto de estudio.

\section{Construcción del prototipo inicial del Modelo Metodológico de Diseño de} SPS .

El desarrollo del prototipo inicial consiste en la caracterización de 15 módulos de un "lienzo de diseño" construido a partir de cinco etapas del proceso de pensamiento de diseño, interpoladas con las tres dimensiones de diseño de un sistema de producto-servicio (figura 3).

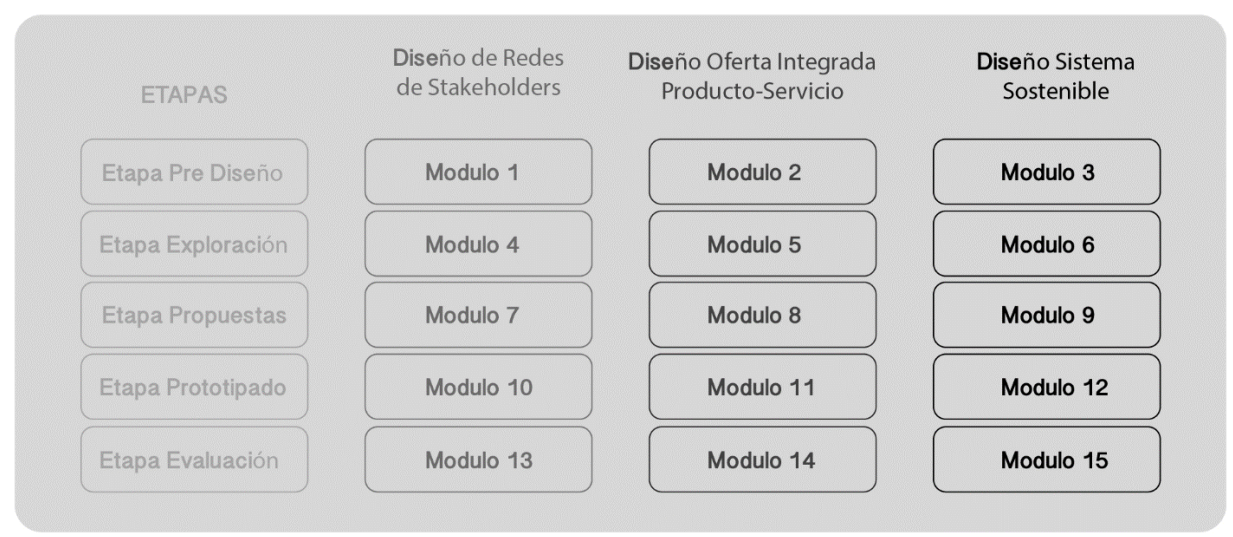

Figura 3. Lienzo base de la creación del prototipo del M.M.SPS. 
El proceso de construcción consistió en caracterizar cada uno de los quince módulos, con base en los factores críticos de implementación identificados, las metodologías con mayor ajuste para implementación en el contexto empresarial de Colombia encontradas, los conceptos de la disciplina del diseño y las condiciones del contexto empresarial estudiado.
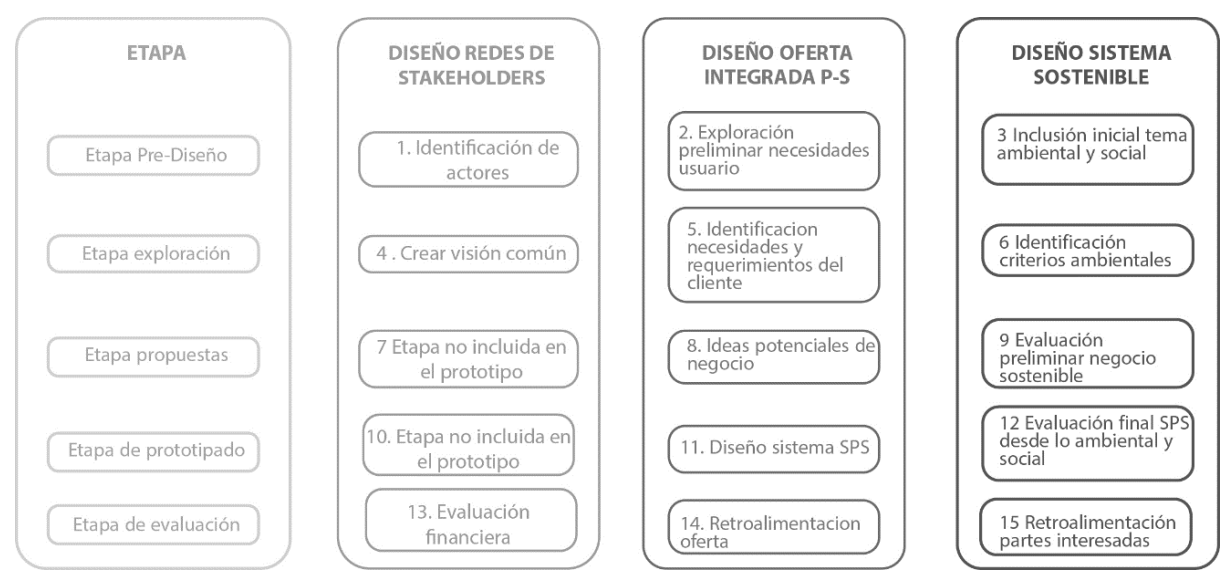

Figura 4. Prototipo inicial del Modelo Metodológico de Aproximación a los M.M.SPS.

Segunda Fase: Fase de investigación en campo

Con el objetivo de validar el prototipo inicial del M.M.SPS, el trabajo de campo consistió en la implementación de tres casos de estudio, la documentación de un caso exitoso y la elaboración de 12 entrevistas con el fin de desarrollar un ejercicio de triangulación de la información que permitiera dar solidez y confiabilidad al modelo propuesto. 


\section{Casos de estudio.}

Los casos de estudio fueron fundamentales para definir cada etapa del modelo, con un enfoque práctico en relación con la implementación, y un enfoque pragmático con respecto a las capacidades y el contexto estudiado. Se escogieron con criterios teóricos, no estadísticos, con el objetivo de repetir hallazgos y extenderlos a la construcción de la teoría propuesta en la investigación. Acorde a los actores involucrados en una solución SPS (empresas, estado, usuarios) y a sus ejes conceptuales: económico, social y ambiental: se decidió trabajar con la principal entidad del Estado en temas ambientales para la ciudad de Bogotá: Secretaria Distrital de Ambiente (SDA) División Empresarial y con su red de empresas ambientales, entidad referente para el país en temas ambientales.

Para cada uno de los casos se siguió la metodología de caso de estudio propuesta por Yin (2014), y se diseñó un protocolo con las siguientes etapas: diseño, preparación, recolección de datos, análisis y conclusiones. La etapa de planeación de cada caso incluyó los criterios arrojados en las conclusiones del caso anterior; de esta manera, se obtuvieron cuatro prototipos del modelo que se fueron ajustando para obtener el modelo final. (Tabla 2)

\section{Entrevistas.}

Con el objetivo de realizar un ejercicio de triangulación de información, se hicieron 12 entrevistas con los participantes de los casos de estudio, el actor gobierno y expertos del tema de sistema producto-servicio y diseño. Para cada actor se elaboró un cuestionario base, con preguntas abiertas pero centradas en el rol de cada participante en el proyecto. Las entrevistas se enfocaron en validar los $\mathrm{FCl}$ e identificar con los participantes su percepción y aprendizaje en el proyecto, su nivel de apropiación de la metodología y sus aportes para ajuste y complementación del prototipo metodológico. Las entrevistas fueron presenciales y semiestructuradas. (Tabla 3) 
Mahecha, N. y Boada, A. / Modelo metodológico de diseño de sistemas producto-servicio (M.M.SPS) en el contexto empresarial colombiano. Una investigación desde el diseño.

Tabla 2. Descripción de casos de estudio.

\begin{tabular}{|c|c|c|c|c|}
\hline Caso de estudio & Perfil & Empresas & $\begin{array}{l}\text { Sistemas producto- servicio } \\
\text { diseñado }\end{array}$ & $\begin{array}{l}\text { Método de } \\
\text { recolección de } \\
\text { información }\end{array}$ \\
\hline $\begin{array}{l}\text { Valorización de } \\
\text { carretes } \\
\text { (caso de éxito) }\end{array}$ & $\begin{array}{l}\text { Alto compromiso } \\
\text { ambiental }\end{array}$ & Procables & $\begin{array}{l}\text { Servicio de valorización de } \\
\text { carretes }\end{array}$ & $\begin{array}{l}\text { Documentación } \\
\text { de entrevistas }\end{array}$ \\
\hline $\begin{array}{l}\text { Movilidad } \\
\text { sostenible }\end{array}$ & $\begin{array}{l}\text { Red de empresas } \\
\text { ambientales. } \\
\text { Secretaría Distrital de } \\
\text { Ambiente. Bogotá }\end{array}$ & $\begin{array}{l}\text { Biomax } \\
\text { SI } 99 \\
\text { Confipetrol } \\
\text { Helm } \\
\text { Procables } \\
\text { PMI }\end{array}$ & $\begin{array}{l}\text { Servicio de transporte privado } \\
\text { empresarial }\end{array}$ & $\begin{array}{l}\text { Observación } \\
\text { participativa } \\
\text { Entrevistas }\end{array}$ \\
\hline $\begin{array}{l}\text { Valorización de } \\
\text { envases }\end{array}$ & & $\begin{array}{l}\text { ECSI } \\
\text { Biomax } \\
\text { Procables }\end{array}$ & $\begin{array}{l}\text { Servicio para la valorización } \\
\text { de envases de lubricantes }\end{array}$ & \\
\hline $\begin{array}{l}\text { Eficiencia } \\
\text { energética }\end{array}$ & $\begin{array}{l}\text { Alto nivel de } \\
\text { innovación y negocios } \\
\text { complementarios }\end{array}$ & $\begin{array}{l}\text { Codensa } \\
\text { Procables }\end{array}$ & $\begin{array}{l}\text { Servicio para la valorización } \\
\text { de cables }\end{array}$ & \\
\hline
\end{tabular}

Nota: Elaboración propia. Descripción de empresas y SPS desarrollados en la etapa de investigación de campo.

Tabla 3. Entrevistas.

\begin{tabular}{|c|c|c|}
\hline Perfil de entrevistados & Número de entrevistas & Perfil \\
\hline Empresa participante & 4 & $\begin{array}{l}\text { Ingenieros ambientales } \\
\text { Administradores } \\
\text { Ingenieros industriales } \\
\text { Líder de compras }\end{array}$ \\
\hline Experto en diseño & 3 & $\begin{array}{l}\text { Diseñador industrial } \\
\text { Ph.D en diseño (2) }\end{array}$ \\
\hline $\begin{array}{l}\text { Experto en sistemas producto- } \\
\text { servicio }\end{array}$ & 2 & $\begin{array}{l}\text { Experto en sostenibilidad y gobierno. } \\
\text { Experto en sistemas producto-servicio }\end{array}$ \\
\hline Actor Gobierno & 3 & $\begin{array}{l}\text { Especialista ambiental } \\
\text { Coordinadores del Programa de Excelencia } \\
\text { Ambiental Bogotá }\end{array}$ \\
\hline Total general & 12 & \\
\hline
\end{tabular}




\section{Análisis de datos}

El análisis de datos consistió en la identificación de patrones comunes de la información recolectada y la triangulación de información con los datos obtenidos a partir de los instrumentos utilizados (casos de estudio-entrevistascaso exitoso), así como la triangulación de información con teoría.

El análisis de información afín con el nivel de apropiación del M.M.SPS se extrae de los datos arrojados por el instrumento de validación del modelo. Esto tuvo como objetivo la confirmación, eliminación o inclusión de nuevas etapas metodológicas en el prototipo teórico, acorde con el contexto de los casos de estudio. Se analizó cada caso de estudio y se incluyeron las conclusiones en el ajuste del M.M.SPS para los siguientes casos. El modelo final con ajustes de casos se contrastó con los resultados de las entrevistas y con el caso de estudio exitoso.

\section{Modelo metodológico de aproximación a los sistemas producto-servicio (M.M.SPS)}

El principal resultado de la investigación es el M.M.SPS, elaborado a lo largo de cinco años de investigación teórica y de campo. Este modelo proporciona una metodología práctica y contextualizada para el diseño de negocios sostenibles, basados en el concepto de sistema producto-servicio en el contexto empresarial colombiano.

El M.M.SPS implica cuatro momentos prácticos fundamentales: análisis estratégico, diseño de la oferta integrada, un momento de análisis de sostenibilidad y, finalmente, estados de evaluación que buscan asegurar en la solución SPS los valores económico, ambiental y social. A continuación, se presenta el modelo final (M.M.SPS). 


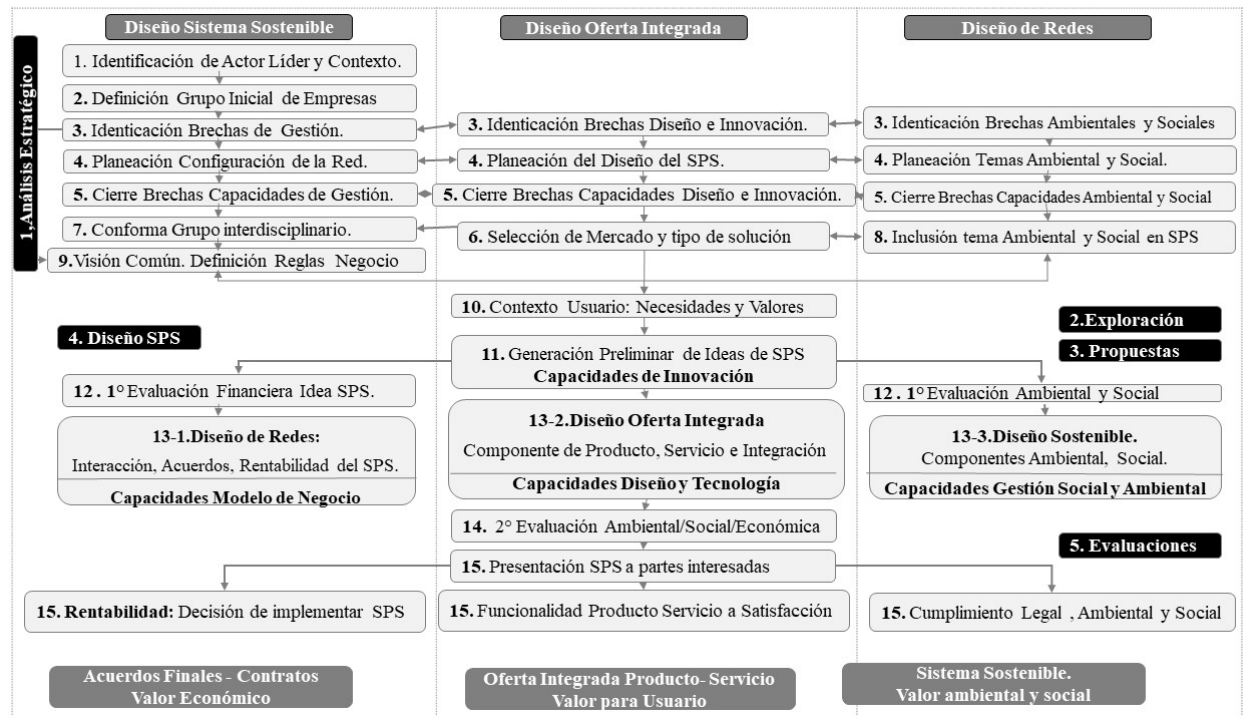

Figura 5. Modelo Metodológico de Aproximación a los Sistemas Producto-Servicio (M.M.SPS) final.

\section{Momento de análisis estratégico}

La etapa de análisis estratégico se propone como una nueva etapa de diseño dentro del modelo (M.M.SPS), que no está incluida en las metodologías teóricas estudiadas. La validación del prototipo en los tres casos de estudio identificó la necesidad de definir criterios de selección de empresas y de alianzas, asegurar el compromiso de los líderes empresariales, garantizar un mínimo común de capacidades relacionadas con gestión administrativa e innovación, diseño, marketing, gestión ambiental y social, asegurar la confianza y visión común, y soportar la identificación del área de negocio. En esta etapa se propone un ejercicio de planeación de recursos, conocimiento, metodología, herramientas 
que se van a utilizar en las etapas posteriores del modelo. La necesidad de estas actividades confirma los factores críticos de implementación de los SPS en países emergentes: falta de herramientas y métodos de diseño, capacidades de emprendimiento y experiencia en la gestión del proceso de servicio (UNEP, 2002).

\section{Diseño de oferta integrada (producto-servicio)}

La etapa de diseño de la oferta debe asegurar la integración de la oferta producto-servicio, vinculando criterios económicos, tecnológicos, ambientales y sociales; además, debe definir las interacciones de la red de actores más eficientes para la solución, y asegurar la satisfacción de las necesidades del usuario y su contexto. El diseño del SPS comprende:

Elementos diseño-producto: 1) Inclusión de los aspectos ambientales en el producto a través de ecodiseño y optimización del ciclo de vida del producto. 2) Inclusión de aspectos culturales y de comportamiento del usuario y de sostenibilidad.

Elementos del servicio: 1) Contenido del servicio. 2) Infraestructura de prestación del servicio. 3) Interfaz con el usuario.

Elementos de oferta integrada: Analizar redundancias entre servicio y producto, alinear flujos de producto y servicio, integrar criterios de diseño a la oferta integrada, función principal y funcionalidades secundarias, interfaz con el usuario y con la red de actores.

Elementos de diseño de la red (acuerdos y contratos): Diseño de flujos e interacciones entre actores requeridos para entregar la oferta integrada al cliente final, según los criterios de sostenibilidad definidos en la etapa de análisis 
estratégico. Implica la definición de reglas contractuales con las cuales se va a regir el nuevo negocio, las responsabilidades y aportes económicos y técnicos de cada actor y el proceso legal de la constitución de un nuevo negocio.

\section{Momento de análisis de sostenibilidad}

Este momento implica definir claramente, en la etapa de análisis estratégico, los criterios ambientales y sociales que hay que cumplir, desarrollando los ajustes requeridos en la etapa de diseño, tales como cierre de brechas de conocimiento, y definición de herramientas ambientales y sociales. Esta actividad exige utilizar principios y herramientas de ecodiseño, diseño de ciclo de vida, principios de economía circular y responsabilidad social en el diseño del producto y servicio.

\section{Etapa de evaluación}

Hacer una evaluación interdisciplinar de valor generado para cada parte interesada de la solución, conforme a criterios establecidos: evaluar la satisfacción de actores de la red (valor económico), usuario y actores (valor social), partes afectadas (valor ambiental y social) y realizar ajustes al diseño final, si se requiere. La valoración de criterios ambientales y sociales exige el conocimiento específico y técnico-legal de la oferta, contexto y usuario; la traducción de dicho conocimiento en criterios de diseño; la identificación de herramientas de diseño de estos criterios y, por último, el reto más sustancial, lograr la aceptación de la propuesta sostenible por parte de la red de actores que terminará en los acuerdos y contratos requeridos para el éxito de la solución sistema producto-servicio.

\section{Discusión}

A continuación, se describen los principales hallazgos encontrados desde la práctica, que obedecen a las condiciones empresariales de un país emergente 
como Colombia. Son particulares del contexto de estudio y no se encuentran en la bibliografía revisada de factores o barreras de éxito de los SPS. Su inclusión en el abordaje de modelos de negocio basados en los SPS en el contexto empresarial de Colombia se hace necesaria y constituye un aporte de esta investigación.

Hallazgo 1. La red de empresas: complementariedad y confianza. En los casos de estudio se identificó la necesidad de complementariedad y confianza en los negocios. El actor líder que inicie la solución debe tener conocimientos explícitos en negocios bajo el concepto SPS, y en la problemática socioambiental desde lo legal y lo técnico. El grupo de empresas que emprendan el negocio deben tener visiones comunes de lo que significa desarrollar un negocio económico, ambiental y social, no ser competidores, tener afinidad de negocios, así como capacidad de asociarse y trabajar bajo acuerdos legales.

De manera transversal al desarrollo de cada etapa, se debe trabajar en la generación de confianza en la red, factor determinante en los acuerdos y contratos que darán viabilidad a la solución. En la generación de confianza es vital asegurar el tema de equidad y capacidad de toma de decisiones de las partes. Hallazgo considerado en las actividades del modelo: 1, 2, 4 y 9.

Hallazgo 2. El involucramiento de la alta gerencia es definitivo para posibilitar el desarrollo de las soluciones SPS. La investigación confirma que el involucramiento gerencial se constituye en un factor crítico para posibilitar el desarrollo de las soluciones SPS. La implementación del SPS es una decisión que se basa más en el nivel de riesgo, emprendimiento e innovación del nivel directivo, que en una decisión técnica o de diseño. Dentro del contexto de gestión empresarial de Colombia es aún más relevante, dado que la mayoría de las empresas son pequeñas y medianas (96\%), con administración tradicional, donde las decisiones se centralizan en la gerencia y son ejecutadas de manera vertical. Hallazgo considerado en las actividades del modelo: 1, 2, 4, 7 y 9. 
Hallazgo 3. Se requieren competencias y conocimientos mínimos para desarrollar con éxito una solución SPS. En los casos de estudio se identificó la falta de conocimiento mínimo en diseño, innovación y gestión ambiental como una barrera en países emergentes (UNEP, 2002). El conocimiento relacionado con negocios sostenibles es insuficiente tanto para los empresarios como para autoridades y profesionales. Los temas débiles encontrados son los siguientes: no se asocia lo ambiental con los negocios, desconocimiento de herramientas de gestión de negocios sostenibles, necesidad de capacidades de diseño, logística inversa, tecnologías de información y reciclaje, y ciclos de vida. El modelo hace un diagnóstico de capacidades de diseño, innovación, gestión, conocimientos ambientales y sociales del grupo de empresas, y despliega un plan para el cierre de las brechas a través de formaciones, contrataciones, inclusión de nuevas empresas y soporte del Estado. Hallazgo considerado en las actividades del modelo: 3 y 5 .

Hallazgo 4. Capacidad de innovación como condición indispensable de implementación de las soluciones SPS. Por medio del trabajo de campo se confirmó el alto nivel de exposición a situaciones nuevas a que se ven abocadas las organizaciones cuando se enfrentan a desarrollar un sistema producto-servicio: asociarse con terceros, involucrarse con el usuario final, hacer cambios en procesos internos, cambiar el producto y servicio ofrecido, tener sistemas de pago diferente, hacer inversiones con grado moderado de incertidumbre, tener una concepción del negocio de manera holística, cumplir criterios ambientales y sociales, entre otros, que hacen de la capacidad de innovación una capacidad obligatoria y no negociable para las organizaciones. En la etapa del modelo se establece el nivel de innovación de las empresas participantes y se elabora un plan para cierre de brechas. Hallazgo considerado en las actividades del modelo: 4 y 5. 
Hallazgo 5. Necesidad de herramientas de diseño y sostenibilidad que faciliten el proceso de desarrollo de los SPS. El desarrollo exitoso del modelo depende de la selección, apropiación y uso acertado de herramientas de diseño y sostenibilidad. Las herramientas se deben aplicar conforme a las capacidades de la red de empresas y su uso debe asegurar el objetivo de la etapa del modelo que soportan. En los casos de estudio se identificó la necesidad de tener herramientas de comunicación, visualización e interacción, identificación de usuario y contexto, diseño de productos y servicios, inclusión y valoración de las variables ambientales y sociales, y surgió la necesidad de una herramienta que soporte el diseño integrado de la solución.

Hallazgo 6. Importancia de una etapa de análisis estratégico para asegurar el éxito de la solución SPS. A diferencia de los modelos aplicados en países desarrollados, en un país emergente como Colombia se requiere una etapa de análisis estratégico debido a que no se cuenta con conocimientos y competencias empresariales maduras ni con un rol protagónico de la disciplina del diseño. Esta etapa asegura las capacidades y recursos mínimos para la viabilidad de la solución SPS, lo que se evidencia en la brecha de capacidades empresariales cuando se buscó integrar lo ambiental y lo social con los negocios. Esto se suma a la complejidad de las soluciones SPS, el nivel de conocimiento, tecnología, diseño e innovación requerido. Hallazgo considerado en las actividades del modelo: 1-9.

Hallazgo 8. Alianza multiempresa. Hay un desconocimiento e incertidumbre jurídica de los empresarios con respecto a las implicaciones legales de una alianza entre organizaciones, situación que se convierte en una aprensión significativa al momento de tomar la decisión de desarrollo del sistema producto-servicio. Por esto se requiere desarrollar herramientas y conocimientos en legislación para soportar a las empresas. Hallazgo considerado en las actividades del modelo: 15. 
Hallazgo 9. Confirmación del rol del Estado. Las políticas públicas enfocadas en incentivar los negocios sostenibles son un factor estructural para lograr su desarrollo, en especial en países emergentes donde la economía de servicio y sostenibilidad aún no se han desarrollado. Mediante las entrevistas y los casos de estudio se pudieron identificar aspectos inherentes al rol del Estado que se deben potencializar: generar micropolítica pública e instrumentos económicos, garantizar la continuidad de los programas que incentiven los negocios sostenibles en cambios de gobierno, asegurar los recursos estatales para la implementación de políticas, fomentar el rol educador del gobierno y potencializar los beneficios de los programas para los empresarios. Hallazgo considerado en las actividades del modelo: 1 y 2 .

\section{Conclusiones}

Durante la fase de construcción teórica y de investigación en campo se evidenció la dificultad de implementar las metodologías existentes, diseñadas para países desarrollados que cuentan con legislaciones sólidas de gestión ambiental y organizaciones con capacidades y conocimientos consolidados de diseño, innovación, sostenibilidad y gerencia, requeridos para el desarrollo de SPS. El modelo metodológico propuesto busca mitigar las barreras de implementación de los SPS y contempla la brecha existente entre capacidades y conocimientos en las organizaciones de países emergentes como Colombia.

Los aportes principales del modelo a la implementación de SPS en contextos emergentes son:

1. Ofrece una metodología concreta y clara para la implementación de una solución SPS en Colombia, a través de la identificación de tres dimensiones definidas y diferenciadas de diseño: diseño de la oferta integrada, de la configuración de la red de empresas y del sistema sostenible. Cada 
dimensión describe las etapas que hay que aplicar dentro del contexto empresarial para asegurar una implementación exitosa y permitir que sean entendidas, comunicadas y evaluadas fácilmente por un equipo interdisciplinario de diseño, especialistas ambientales o administradores.

2. La metodología propuesta cuenta con un enfoque sistémico que aborda todo el ciclo de diseño de un SPS, en contraposición a las metodologías existentes, las cuales se enfocan en etapas particulares, dependiendo de la disciplina de origen, diseño de la oferta, o proceso de manufactura o diseño de la red. El modelo incluye dos etapas no contempladas en otros modelos, tales como el desarrollo legal y contractual de la red y la evaluación permanente, para asegurar el cumplimiento de los criterios económicos, ambientales y sociales.

3. El seguimiento de las etapas del M.M.SPS asegura en un alto porcentaje una implementación exitosa de la solución SPS en el contexto colombiano, debido a la rigurosa metodología utilizada para la definición de las quince etapas. En esta se tomaron en cuenta las metodologías existentes, los factores críticos de implementación teóricos más reconocidos, y los de contexto identificados en los casos de estudio, todo unido al ejercicio de validación en campo.

4. Los resultados de esta investigación contribuyen al entendimiento de los SPS en economías emergentes y brinda un aporte a la disciplina del diseño a través de la metodología de implementación, con el objetivo de avanzar en la implementación en Colombia a gran escala de las soluciones SPS.

Las futuras investigaciones deben centrarse en:

- Cambio cultural en el paradigma de la propiedad, principal factor crítico de implementación identificado a nivel teórico. Se deben resolver preguntas como: ¿es posible en Colombia pasar de la etapa de consumo individual hacia una economía de servicio basada en la satisfacción y un bajo consumo? ¿Son viables en Colombia conceptos como bienes comunes, bienestar, comunidad? 
- Claridad jurídica. En aras de fortalecer la etapa final de acuerdos entre las partes interesadas, materializadas en contratos con efectos civiles y penales, es necesario investigar sobre herramientas que faciliten la comunicación y confianza de las partes interesadas. Se deben responder preguntas de este tipo: ¿cómo potenciar el factor confianza a lo largo de la implementación del sistema producto-servicio? ¿Cuáles son las configuraciones de la red de partes interesadas con mayor fortaleza en el contexto colombiano? ¿Cómo mejorar y potenciar las capacidades de gestión de la red?

Finalmente, cabe destacar que la discusión sobre el modelo de diseño propuesto sigue abierta; un enfoque metodológico único no es posible, debido al alto margen de incertidumbre de los contextos empresariales en Colombia. Se deben realizar nuevos casos de estudio para su implementación, con miras a mejorar los métodos, herramientas y etapas propuestas.

\section{Referencias}

Aicher, O. (1992). Analógico y digital. Barcelona: Gustavo Gili. ISBN-13: 9788425218460

Baines, T.S., Lightfoot, H., and Steve, E. (2007). State-of-the-art in product service-systems. Part B: Journal of Engineering Manufacture. 221. 15431552. 1-10. DOI: 10.1243/09544054JEM858

Bartelmus, P. (1997). Sustainable Development Paradigm or Paranoia. Environment and Development Economics, 2, 1-9. DOI: 10.1504/ IJSD.2000.001537

Boada, A., and Mont, O. (2005). Desmaterialización de sistemas productoservicio, una estrategia diferente de negocios. Bogotá: Universidad Externado de Colombia. ISBN: 9789586169820 
Boehm, M., and Oliver, T. (2013). Looking beyond the rim of one's teacup: a multidisciplinary literature review of Product-Service Systems in Information Systems, Business Management, and Engineering \& Design. Journal of Cleaner Production, 245-260. DOI: 10.1016/j.jclepro.2013.01.019

Bor, J. (1996). Ecodiseño y desarrollo sostenible. Revista de Coyuntura Económica, 2, 117-129. ISSN 0213-2273

Brezet, J. (2001). The Design of Eco-efficient Services. Design for Sustainability Program. Helsinki: Delft University of Technology.

Buchanan, R. (1992). Wicked Problems in Design Thinking. Design Issues, 5-21. DOI: 10.2307/1511637

Buchanan, R. (2004). Dignidad y derechos humanos. Hacia un diseño centrado en lo humano. Encuadre. Revista de la Enseñanza del Diseño, 2 (3). México.

Buchanan, R., and Margolin, V. (1995). Discovering Design. Chicago: University of Chicago Press. ISBN-13: 978-0226078151

Catulli, M., Lindley, J., and Reed, N. (2013). What is Mine is not Yours: Further Insight on what Access-Based Consumption says about Consumers. Research in Consumer Behavior, 15, 185-208. DOI: 10.1108/S08852111(2013)0000015012

Ceschin, F. (2013). Critical factors for implementing and diffusing sustainable. Journal of Cleaner Production 45, 74-88. DOI: 10.1016/j. jclepro.2012.05.034.

Clayton, R., and Backhouse, C. (2011). Evaluating existing approaches to product-service system design: A comparison with industrial practice. Journal of Manufacturing Technology Management, 23 (3). DOI: 10.1108/17410381211217371 
Comisión Mundial para el Medio Ambiente y el Desarrollo de la ONU (1987). Informe Brundtland. Comisión Mundial para el Medio Ambiente y el Desarrollo de la ONU.

Forty, A. (1986). Objects of desire. Design and society since 1750. Londres: Thames \& Hudson. ISBN-13: 978-0500274125

Glavic, P., and Lukman, R. (2007). Review of sustainability terms and their definitions. Journal of Cleaner Production 15, 1875-1885. https://doi. org/10.1016/j.jclepro.2006.12.006

Goedkoop, M. (1999). Product service systems ecological and economic basics. The Netherlands: Pricewaterhouse Cooper.

Hänsch, F., and Gitirana, M. (2013). Product-service systems: a literature review on integrated products and services. Journal of Cleaner Production. DOI: 10.1016/j.jclepro.2016.08.061

Kanda, Y., and Nakagami, Y. (2006). What is Product-Service Systems (PSS)? Review on PSS Researches and Relevant Policies. Institute for Global Environmental Strategies (IGES), 17.

Krippendorff, K. (2006). The semantic turn. Boca Ratón: Taylor and Francis Group.

Laperche, B., and Picard, F. (2013). Environmental constraints, ProductService Systems development and impacts on innovation management: learning from manufacturing firms in the French context. Journal of Cleaner Production, 53, 118-128. DOI: 10.1016/j.jclepro.2013.03.047

Manzini, E. (2003). Product-Service Systems and Sustainability. Milán: Interdepartmental Research Centre Innovation for the Environmental Sustainability (CIR.IS). 
Manzini, E. (2004). Solution oriented partnership. Bedfordshire, UK: Granfield University.

Manzini, E. (2006). Design, ethics and sustainability. Milán: Politecnico di Milano.

Margolin, V. (1995). The idea of design. Cambridge-Massachusetts, Londres: The MIT Press.

Margolin, V. (2005). Las políticas de lo artificial. Chicago: The University of Chicago Press.

Mont, O. (2002). Clarifying the concept of product service system. J Cleaner Production, 237-245. DOI: 10.1016/S0959-6526(01)00039-7

ONU. (25 de septiembre de 2015). Transformar nuestro mundo: la Agenda 2030 para el Desarrollo Sostenible. Resolución aprobada por la Asamblea General el 25 de septiembre de 2015.

Pezzotta, G., Cavalieri, S., and Shimomura, Y. (2013). A service engineering framework to design and configure Product-Service Systems. 11th IFAC Workshop on Intelligent Manufacturing Systems, 22-24. DOI: 10.13140/ RG.2.1.4983.0247

PNUMA \& WWF. (1991). Cuidar la Tierra. Estrategia para el futuro de la vida.

Reim, W., Parida, V., and Örtqvist, D. (2015). Product-Service Systems (PSS) business models and tactics: a systematic literature review. Journal of Cleaner Production, 61-75. DOI: 10.1016/j.jclepro.2014.07.003

Rocchi, S. (2005). Enhancing sustainable innovation by design. Rotterdam: Erasmus University Rotterdam.

Schenkl, S., Rösch, C., and Mörtl, M. (2014). Literature study on factors influencing the market acceptance of PSS. ScienceDirect, 98-103. DOI: 10.1016/j.procir.2014.01.013

Sen, A. (2000). Desarrollo y libertad. Barcelona: Editorial Planeta. 
Tsai, C.K., and Hsin, Y.M. (2010). Barrier analysis for product service system using interpretive structural model. Int J Adv Manuf Technol, 49: 407-417. DOI: $10.1007 / \mathrm{s} 00170-009-2399-7$

Tukker, A. (2004). Eight types of product-service system: eight ways to sustainability? Business Strategy and the Environment, 246-260. DOI: $10.1002 /$ bse.414

Tukker, A. (2006). Product-services as a research field: past, present and future. Reflections from a decade of research. Journal of Cleaner Production, 14, 1552-1556. DOI: 10.1016/j.jclepro.2006.01.022

Tukker, A. (2013). Product services for a resource-efficient and circular economy: a review. Journal of Cleaner Production, 1-16. DOI: 10.1016/j. jclepro.2013.11.049

UNEP, U.N. (2002). Product-ServiceSystems and Sustainability. En Opportunities for Sustainable Solutions. París: UNEP, Division of Technology Industry and Economics, Production and Consumption Branch.

Vasantha, G., Rajkumar, R., Lelah, A., and Brissaud, D. (2012). A review of product-service systems design. Journal of Engineering Design, 635-659. DOI: $10.1080 / 09544828.2011 .639712$

Vezzoli, C., and Ceschin, F. (2012). Why have "Sustainable Product-Service Systems" not been widely implemented? Journal of Cleaner Production, 288-290. DOI: 10.1016/j.jclepro.2012.05.050

Vezzoli, C., and Manzini, E. (2008). Design for Environmental Sustainability. Milán: Politecnico di Milano.

Vezzoli, C., Ceschin, F., Diehl, J., and Kohtala, C. (2015). New Design Challenges to Widely Implement 'Sustainable Product-Service Systems. Journal of Cleaner Production, 1-19. DOI: 10.1016/j.jclepro.2015.02.061 
Vezzoli, C., Kohtala, C., and Srinivasan, A. (2014). Product-Service System Design. Sheffield, UK: Published by Greenleaf Publishing Limited. ISBN: 978-1906093-67-9

White, A., Stoughton, M., and Feng, L. (1999). Servicizing: The Quiet Transition to Extended Product Responsibility. Boston: Tellus Institute.

Yin, R.K. (2014). Case Study Research. Design and Methods. Washington, DC: Sage Publications.

Como citar: Mahecha, N. y Boada, A. (2020). Modelo metodológico de diseño de sistemas producto-servicio (M.M.SPS) en el contexto empresarial colombiano. Una investigación desde el diseño. Revista KEPES, 17 (21), 459-492. DOI: 10.17151/kepes.2020.17.21.16 